ACCEPTED MANUSCRIPT

\title{
Arrays of suspended silicon nanowires defined by ion beam implantation: mechanical coupling and combination with CMOS technology
}

To cite this article before publication: Jordi Llobet et al 2018 Nanotechnology in press https://doi.org/10.1088/1361-6528/aaac67

\section{Manuscript version: Accepted Manuscript}

Accepted Manuscript is "the version of the article accepted for publication including all changes made as a result of the peer review process, and which may also include the addition to the article by IOP Publishing of a header, an article ID, a cover sheet and/or an 'Accepted Manuscript' watermark, but excluding any other editing, typesetting or other changes made by IOP Publishing and/or its licensors"

This Accepted Manuscript is (C) 2018 IOP Publishing Ltd.

During the embargo period (the 12 month period from the publication of the Version of Record of this article), the Accepted Manuscript is fully protected by copyright and cannot be reused or reposted elsewhere.

As the Version of Record of this article is going to be / has been published on a subscription basis, this Accepted Manuscript is available for reuse under a CC BY-NC-ND 3.0 licence after the 12 month embargo period.

After the embargo period, everyone is permitted to use copy and redistribute this article for non-commercial purposes only, provided that they adhere to all the terms of the licence https://creativecommons.org/licences/by-nc-nd/3.0

Although reasonable endeavours have been taken to obtain all necessary permissions from third parties to include their copyrighted content within this article, their full citation and copyright line may not be present in this Accepted Manuscript version. Before using any content from this article, please refer to the Version of Record on IOPscience once published for full citation and copyright details, as permissions will likely be required. All third party content is fully copyright protected, unless specifically stated otherwise in the figure caption in the Version of Record.

View the article online for updates and enhancements. 


\title{
Arrays of suspended silicon nanowires defined by ion beam implantation: mechanical coupling and combination with CMOS technology
}

\author{
J. Llobet, ${ }^{\mathrm{a}, \mathrm{b}}$ G. Rius, ${ }^{\mathrm{a}}$ A. Chuquitarqui, ${ }^{\mathrm{a}}$ X. Borrisé, ${ }^{\mathrm{c}}$ R. Koops, ${ }^{\mathrm{d}}$ \\ M. van Veghel, ${ }^{d}$ and F. Perez-Murano ${ }^{a}$ \\ ${ }^{a}$ Institute of Microelectronics of Barcelona (IMB-CNM, CSIC), 08193 Bellaterra, Spain \\ ${ }^{\mathrm{b}}$ International Iberian Nanotechnology Laboratory (INL), 4715-330 Braga, Portugal \\ ${ }^{c}$ Catalan Institute of Nanoscience and Nanotechnology (ICN2), \& The Barcelona Institute of \\ Science and Technology (BIST), 08193 Bellaterra, Spain \\ ${ }^{d}$ VSL Dutch Metrology Institute, Thijsseweg 11, 2629 JA Delft, The Netherlands
}

\begin{abstract}
We present the fabrication, operation, and CMOS integration of arrays of suspended silicon nanowires (SiNWs). The functional structures are obtained by a top-down fabrication approach consisting in a resistless process based on focused ion beam irradiation, causing local gallium implantation and silicon amorphization, plus selective silicon etching by tetramethylammonium hydroxide, and a thermal annealing process in a boron rich atmosphere. The last step enables the electrical functionality of the irradiated material. Doubly clamped silicon beams are fabricated by this method. The electrical readout of their mechanical response can be addressed by a frequency down-mixing detection technique thanks to an enhanced piezoresistive transduction mechanism. Three specific aspects are discussed: i) the engineering of mechanically coupled SiNWs, by making use of the nanometer scale overhang that it is inherently-generated with this fabrication process, ii) the statistical distribution of patterned lateral dimensions when fabricating large arrays of identical devices, and iii) the compatibility of the patterning methodology with CMOS circuits. Our results suggest that the application of this method to the integration of large arrays of suspended SiNWs with CMOS circuitry is interesting in view of applications such as advanced radio frequency band pass filters and ultra-high-sensitivity mass sensors.
\end{abstract}




\section{Introduction}

Microelectromechanical systems (MEMS) and nanoelectromechanical systems (NEMS)/are miniaturized devices that transduce mechanical energy into electrical energy or vice versa. MEMS and NEMS usually can operate as sensors or actuators, i.e. they can be used as interfaces that sense or interact with the physical world [1].

Suspended NEMS, such as based on either nanowires (NWs) [2] or nanotubes [3], have been studied and demonstrated their advanced capabilities in several fields. For instance, for telecommunications, NEMS allow adopting solutions for integrated oscillators [4,5] or high frequency electrical-mechanical filters [6,7]. Among their potential functionalities, NEMS can be used as transistors or mechanical switches and logics [8]. Particularly, doubly clamped, suspended NW or nanotube structures present exceptional properties for nanomechanical resonator electronic applications [2,9-11]. As sensors, when their dimensions are reduced their mass sensitivity is highly increased, so that ultra-precise detectors can be obtained [12-17]. NWs have shown excellent performance as building blocks in fields such as chemical sensing $[18,19]$ and force sensing [20].

Large efforts for fabricating NW devices have been made on several materials [21]. SiNWs are of special interest thanks to their convenient electromechanical properties and their potential compatibility with mainstream microelectronics technologies, provided that proper fabrication methods for integrating the functional nanostructures are developed [22]. Silicon is a semiconductor material and has piezoresistive properties [23] two physical characteristics which are particularly interesting for sensing applications. A number of approaches have been reported in the literature to engineer nanomechanical structures. They are typically based on either top-down (high resolution lithography plus pattern transfer) or bottom-up (e.g. catalytic growth) methods, but can also be attained as a combination of both $[11,22]$. Bottom-up methods face difficulties of placement accuracy and yield and they face limitation in terms of design flexibility. Differently, top-down methods typically use electron beam lithography (EBL) to define SiNWs with submicron precision plus at least one additional lithographic step for defining the electrical contacts. Among other limitations of top-down approach, obtaining suspended Si structures of different thicknesses is not straightforward and the fabrication process is costly. As an alternative, ion implantation by a focused ion beam (FIB) plus wet etching [24] and thermal annealing allows the fabrication of suspended beams that are functional from the electrical and mechanical point of view, yet without using any resist [25]. This method is especially simple, which makes it suitable for prototyping, as only three single processing steps are needed, compared with a minimum of seven single processes steps required for EBL. Additional advantages provided by this method are the fact that self-under-etching occurs for narrow structures as the final thickness of the nanowire is dictated by the ion implantation range, and a potential lower proximity effect.

A functional resonator based on suspended SiNWs requires actuation and detection of the beam oscillations. This is achieved by electrostatic actuation and piezoresistive detection. The piezoresistance is quantified by the gauge factor $(\mathrm{G}), \mathrm{G}=\left(\Delta \mathrm{R} / \mathrm{R}_{0}\right) /\left(\Delta \mathrm{l} / \mathrm{l}_{0}\right)$, that is, the variation of electrical resistance $\left(\Delta R / R_{0}\right)$ as a function of the elongation of the device $\left(\Delta \mathrm{l} / \mathrm{l}_{0}\right)$. The electrical detection of the oscillation is performed by a down-mixing radio-frequency detection method, in which the high frequencytransduced signal arising from the motion of the resonator is transferred to lower frequencies, and then detected using a lock-in amplifier. As it is demonstrated in our previous work [2], for small vibrations $\left(\mathrm{a}_{\mathrm{n}}\right)$ and pre-strained devices $\left(\mathrm{d}_{0}\right)$, the transduction is enhanced by the presence of asymmetries in the SiNW. The 2-sources - 1 frequency detection method used in this present work provides an electrical read-out based on this linear transduction mechanism, as proven before [26].

Here, we explore three aspects to advance in the applicability of this fabrication method in view of developing practical resonating nanomechanical devices: the operation of mechanically coupled nanowires, the fabrication of large arrays of SiNWs, and their combination with CMOS circuit fabrication. 
The SiNWs fabrication is realized on a silicon on insulator (SOI) substrates. The SOI layer has a (110) orientation, a nominal thickness of $2 \mu \mathrm{m}+/-0.5 \mu \mathrm{m}$ and an initial resistivity of around $10 \Omega \cdot \mathrm{cm}$ (boron doping). The thickness of the buried oxide is $2 \mu \mathrm{m}$. A focused $\mathrm{Ga}^{+}$beam $(30 \mathrm{keV})$ is used as a lithography method as described elsewhere [24,25,27-29]. The ion exposure of a crystalline surface to an energetic focused beam causes the controlled implantation of those atoms and induces local amorphization of the target crystalline material [30-34], as corroborated by HR-TEM imaging and Raman spectroscopy [25] Remarkably, the modified volume is highly resistant to silicon etchants and therefore, it can be used as a robust pattern-transfer mask. [23]

The effect on ion irradiation and implantation on solid substrates has been widely studied [35]. At low doses, the main effect of $\mathrm{Ga}^{+}$irradiation of silicon is the implantation of atomic $\mathrm{Ga}$ and the amorphization of silicon crystal due to an energy transfer phenomenon. At the range of energies typically used in FIB processes (tens of $\mathrm{keV}$ ), it is agreed that the dominant mechanism of energy exchange involves elastic interaction between the incident ion and the target ion (nuclear stopping). Experimental results indicate that typical ion dose for achieving an etchant-resistant layer is $\sim 10^{16}$ ion $/ \mathrm{cm}^{2}$. This dose is sufficiently low to avoid relevant surface milling [36].

A $30 \mathrm{keV}$ ion energy has been used in all the experiments reported in this work, which is the maximum energy available in our FIB system. Implanted volume is related with the scattering range of the incident ions delivered to the target substrate: for $30 \mathrm{keV}$, the longitudinal ion range of $\mathrm{Ga}^{+}$in silicon at $0^{\circ}$ (perpendicular incidence) is $28.6 \mathrm{~nm}$ and the radial ion range is $11.6 \mathrm{~nm}$. At lower beam energy $(5 \mathrm{keV})$ they become $8.7 \mathrm{~nm}$ and $3.6 \mathrm{~nm}$, respectively. [35] It is reported that the final dimensions of the nanowires roughly correspond to the dimensions of the Ga implanted volume.[25]

Specifically, anisotropic wet silicon etching by tetramethylammonium hydroxide (TMAH) at $25 \%$ concentration and $80^{\circ} \mathrm{C}$ has been used. TMAH etching solution removes the silicon volume that is not modified by the ion implantation. Noteworthy, the depth of the amorphization is directly proportional to the ions energy [37,38], i.e. it opens the possibility the fabrication of devices of different thicknesses with single nanometer scale precision.

By means of a smart design, three dimensional, complex nanostructures can be easily engineered by this method. Anisotropic wet etching permits to generate suspended or not suspended structures by selecting the orientation of the layout according to the etching rate of the silicon plane [39]. Moreover, combining ion implantation and milling, it is also possible to define particular three dimensional structures as, for example, functional purpose-oriented supporting micro-posts [40]. As it will be shown later in this report, the supporting micro-posts are used to finalize the fabrication of the devices.

The electrical resistance of crystalline silicon nanowires is mainly dictated by their doping level and dimensions, theoretically according to Ohm's law. For the nanowires depicted in figure 1, the experimental resistance value is typically of tens of $\mathrm{k} \Omega$. Additional effects must be considered particularly for silicon nanowires of very small dimensions. A reduction of the nanowire diameter implies that the presence/amount of surface charges induces the charge depletion of silicon and, consequently, an increase of resistance.

For the silicon nanowires fabricated by FIB ion implantation, it is found that as a result of the ion-beam induced amorphization of $\mathrm{Si}$, the modified material becomes highly insulating. However, we have previously shown [25] that the degradation of electrical properties can be compensated by a thermal annealing at, for example, a temperature $>600{ }^{\circ} \mathrm{C}$ in a boron-rich environment. The high temperature annealing allows to partially recover the Si crystallinity and to eliminate the gallium from the nanowire. The annealing is performed in conjunction with doping, which is necessary to increase the conductivity of the nanowires. Specifically, considering the base doping level of the SOI layer, the resistance of the 
silicon nanowires depicted in figure 1 would have been of more than $5 \mathrm{M} \Omega$, which would preclude the electrical read-out. The typical resistivity of the nanowires after the annealing and doping step is 0.02 $\Omega \cdot \mathrm{cm}$. Additionally, due to the formation of silicon nanocrystals during the annealing, single electron effects can be observed, which provides additional value and potential to present fabrication method [41].

The electrical contact pads and lines are made also of silicon, and they are defined at the same time (same exposure) than the suspended nanowires, taking advantage of the fact that large implanted areas do not become fully under-etched. In the supplemental information, more information about the complete device lay-out and bonding is presented.

\section{Results and discussion}

\subsection{Coupled operation of suspended silicon nanowires}

We have previously studied the electromechanical performance of doubly clamped resonating devices fabricated by FIB implantation on silicon [26]. A characteristic feature of this fabrication process is that a thin overhang is formed along the edge of the resonator clamping areas. The width of the overhang is dependent on the etching time in combination with the orientation of the edge of the clamping area with respect to the Si crystalline plane $[25,40]$. Here, we actually take advantage of the overhang formation to generate coupled mechanical resonator systems based on a pair of doubly clamped SiNWs and demonstrate their coupled mechanical behaviour. Coupling of suspended SiNWs has already proven suitable for the realization of ultrasensitive nanomechanical mass sensors [17,42]. In that work [42], optical lithography methods were used. Also distinctive in terms of fabrication, in another work [17], a single clamped SiNW was grown at the edge of a single clamped micrometric cantilever, i.e. based on a bottom-up approach.

In Fig. 1, three different pairs of coupled SiNWs operated as mechanical resonators are shown. The separation between the beams can be precisely controlled, as clearly observed in the SEM images (Fig. 1 (a-c)). Respectively, in Fig.1 (d-f) the mechanical response of each nanoresonator system is displayed. As mentioned, the experimental results of their electromechanical response are obtained by electrical readout using the frequency down-mixing detection technique described in some previous works $[2,10]$. Additional information can be found in the supplemental information. The two obtained resonance modes of the coupled SiNWs correspond to the symmetrical (lower frequency) and anti-symmetrical (higher frequency) flexural modes. It is observed that the symmetrical/anti-symmetrical frequency split between the two modes increases inversely with their separation. This behavior indicates that the coupling is larger as the distance between the beams decreases.

Quantitatively, the frequency split effect can be explained by using the ideal coupled harmonic oscillator's theory [42] which holds that if $\mathrm{N}$ resonators are coupled, $\mathrm{N}$ collective modes of oscillation will be generated, where individual resonators will oscillate either in phase or antiphase with respect to their adjacent neighbours. For the particular case of two parallel identical resonators (nanobeams with the same dimensions), the mechanical coupling generates two different vibration modes: symmetric, nanobeams oscillating in phase, and antisymmetric, nanobeams oscillating in antiphase. 


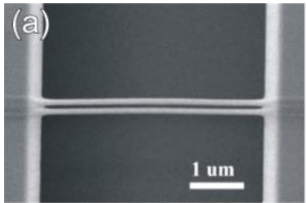

(d)

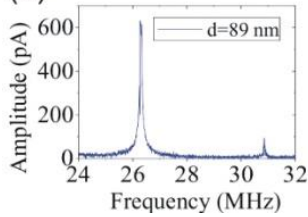

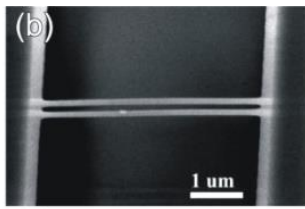

(e)

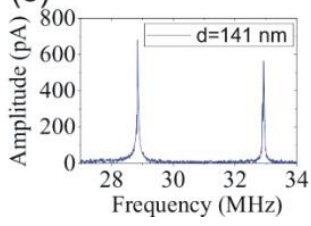

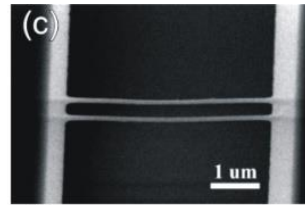

(f)

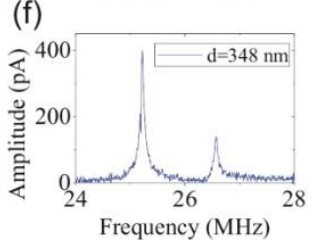

(g)

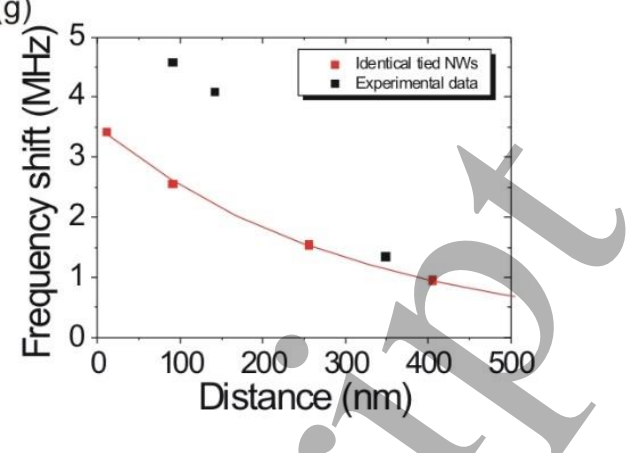

Figure 1. (a to c) SEM images of coupled, parallel SiNW-resonators with different coupling distances, $d$ : (a) $89 \mathrm{~nm},(b) 141 \mathrm{~nm}$, (c) $348 \mathrm{~nm}$. (d to f) Measurements of the symmetric and anti-symmetric resonance modes. The SiNWs have an average length of $4.1 \mu \mathrm{m}$, an average cross-sectional dimension of $40 \mathrm{~nm}$ (thickness) $\times$ $135 \mathrm{~nm}$ (width), and membranes of an average width of $250 \mathrm{~nm} .(\mathrm{g}$ ) Plot of the experimental (black marks) and theoretical (red marks and fit) frequency shifts between the symmetric and the anti-symmetric modes as a function of coupling distances.

The system has been modelled by applying simulations with the COMSOL Multiphysics package. The effect of coupling distances (Fig. 1, (g)) has been investigated. Calculations indicate that the frequency shift decreases when the coupling distance increases, confirming the experimental trend. The discrepancy in the absolute values of the frequency shift between COMSOL simulations and experimental observations can be explained by the uncertainty in the thickness of the coupling membrane. The electromechanically coupled behaviour ean be used for different applications such as radio frequency (RF) filters [43] and ultra-high sensitivity sensors [44]. Specifically, a radio frequency filter can be obtained by the superposition of several resonance peaks, which can be fabricated as multiple parallel resonating beams with coupling distances, d, longer than $1 \mu \mathrm{m}$.

\subsection{Large array of nanowires}

The results shown in figure 1 trigger an interest to evaluate the fabrication using FIB implantation of integrated systems consisting in large arrays of suspended nanowires. An example of the capability of this method is shown in figure 2 . The array consists of more than 3,000 suspended SiNWs of typical dimensions: $2.5 \mu \mathrm{m}$ in length, $100 \mathrm{~nm}$ in width (xy), and $40 \mathrm{~nm}$ thickness (z). The aim was to show that a dense array of suspended silicon nanowires can be fabricated with good reproducibility by the FIB implantation method. As an application, by combining large arrays of almost identical silicon nanowires with optical detection, a large-area nanomechanical mass sensor could be developed. 

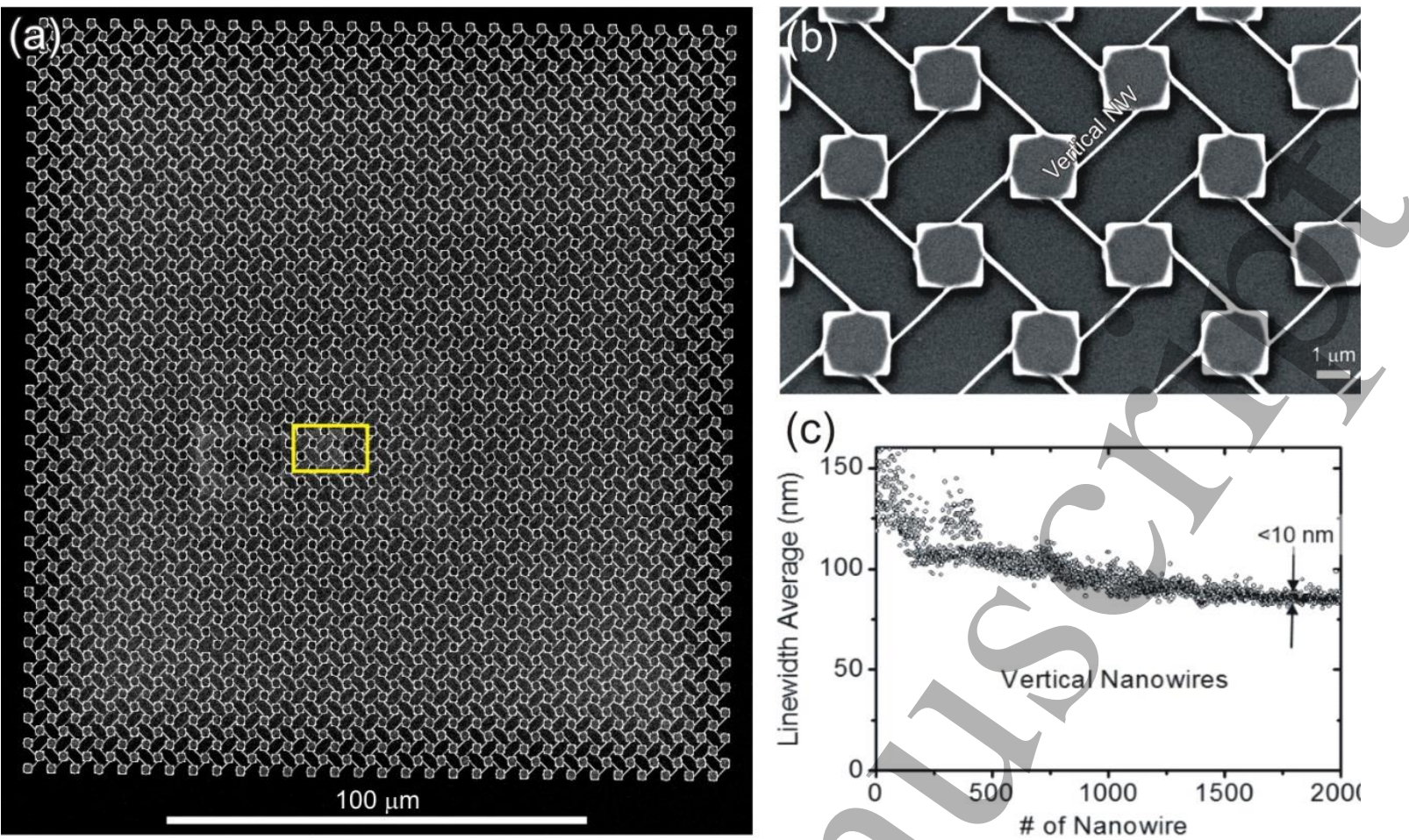

Figure 2. (a) SEM image of a large array of suspended SiNWs covering an area of $180 \times 180$ um $^{2}$ (that is maximum write field for our FIB system) with more than 3000 structures. (b) Zoom-in image of the suspended NWs marked with a yellow square in (a). (c) Distribution of the linewidth average values for the nanowires depicted in (a).

A detailed inspection of the array reveals the absence of missing, broken or collapsed (instead of suspended) SiNWs, which is a measure of the good fabrication yield that can be achieved with this approach. An exhaustive metrological analysis has been performed to determine the patterning accuracy in defining the SiNWs dimensions. In particular, a dedicated method has been established to automatically determine the width and length of every single SiNW in the array. The method consists in collecting a large set of SEM images at high magnification $(8.00 \mathrm{kX})$. Then, the image analysis is automatized. In this case, it is applied separately for the NWs in the two perpendicular directions that they have been oriented in their design. For each type, more than 1500 wires were analysed. The linewidth values obtained for the vertical SiNWs are represented in figure 2 (c). The values are sequentially displayed according to the temporal order in which the respective SEM image were taken.

The statistical data processing allows identifying three different sources of values dispersion: a) a timeinduced variation in recorded length and width of NWs, b) a large variability in width that occurs for the NWs located at the initial part of the array, and lastly, c) intrinsic linewidth statistical dispersion. Effect a) is attributed to an instrumental origin, i.e. an effect of the SEM beam drift due to unbalanced thermal variations that leads to distorted imaging, while b) is ascribed to either variations with the ion beam current during the initial part of the patterning of the array or to the effect of charging due to the dose employed. Finally, c) is considered to be due to intrinsic variability of the fabrication process itself. Accordingly, effects a) and b)/can be ruled out in the analysis of the inherent dispersion in the dimensions due to the fabrication process, since they can be solved by improving SEM imaging procedure, compensating the array design by adjusting the dose or with a proper initialization of the patterning process. Summarizing, we can infer that the value of the statistical dispersion (1-sigma) in SiNWs width attributable to the fabrication process is below $10 \mathrm{~nm}$ (Figure 2). This level of accuracy is of remarkable interest as it allows guaranteeing a precise tuning of the resonating frequency. 


\subsection{Integration with CMOS circuitry}

Control of a large number of suspended nanowires requires their combination with an integrated electronic circuit if, for example, each nanowire, or group of nanowires, needs to be addressed individually. Here, we have investigated aspects related to alignment and circuit induced-damage for the integration of SiNWs as a fabrication process based on the combination of FIB implantation with CMOS technology.

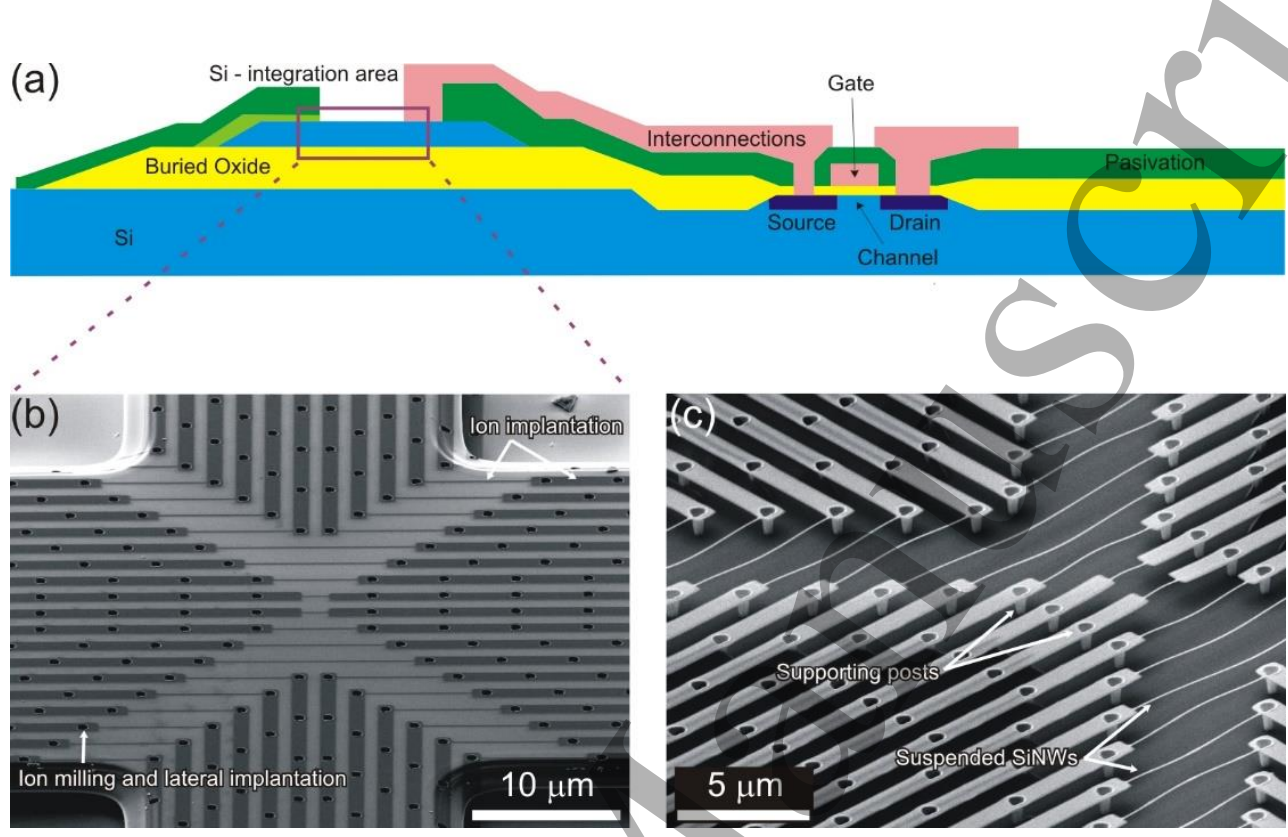

Figure 3. (a) Drawing showing the integration scheme to combine CMOS circuits (a MOS transistor, in the drawing) with an integration area where the FIB nanopattterning is performed. The integration scheme is based on using SOI wafers. (b) SEM image of the integration area taken after the ion beam exposure. (c) SEM image of the same structure than in (b) taken after the wet chemical etching.

The approach for combining FIB implantation and CMOS integration has been addressed as follows. A previously developed SOI-CMOS platform [45] has been used because of its suitability to be combined with nanofabrication processes. Specifically, it is based on the use of SOI substrates, being the nanostructured devices fabricated in the SOI layer while the CMOS circuit is built in the bulk silicon (see figure 3 (a)). First, the areas where the nanopatterning will be applied are covered by a protection layer in order to avoid any surface damage during the fabrication of the CMOS circuit. The unprotected areas are etched down to the bulk silicon, where the CMOS circuits are fabricated. Then, the fabrication of the CMOS circuit is performed. The CMOS circuit technology is the CNM-CMOS technology available in house, which is a simple CMOS technology consisting of two polysilicon levels and one metal level. A particular feature of the CMOS-SOI platform is the way that the electrical contacts between the CMOS and the SOI layers are realized. For this. a specific process had to be developed [45], providing smooth sidewalls for the bulk silicon - SOI layer transition. . At this point, the fabrication of the devices in the nanopatterning area is realized by following the steps described previously (section 2).

Several arrays of suspended nanowires have been fabricated to verify that the process is applicable on a CMOS platform. First, we have analysed the positioning of the array into the area dedicated to the integration. Figure 3 (b), shows the array after the ion beam irradiation. A successful alignment of the device inside the integration area has been achieved. Yet, due to some inaccuracy between the magnification factors of the SEM and the FIB, the area of the array is larger than the available space in 
the integration area. Only because of this, the edges of the array have not been properly defined. An image of the same array after completing the fabrication process is shown in figure 3 (c).
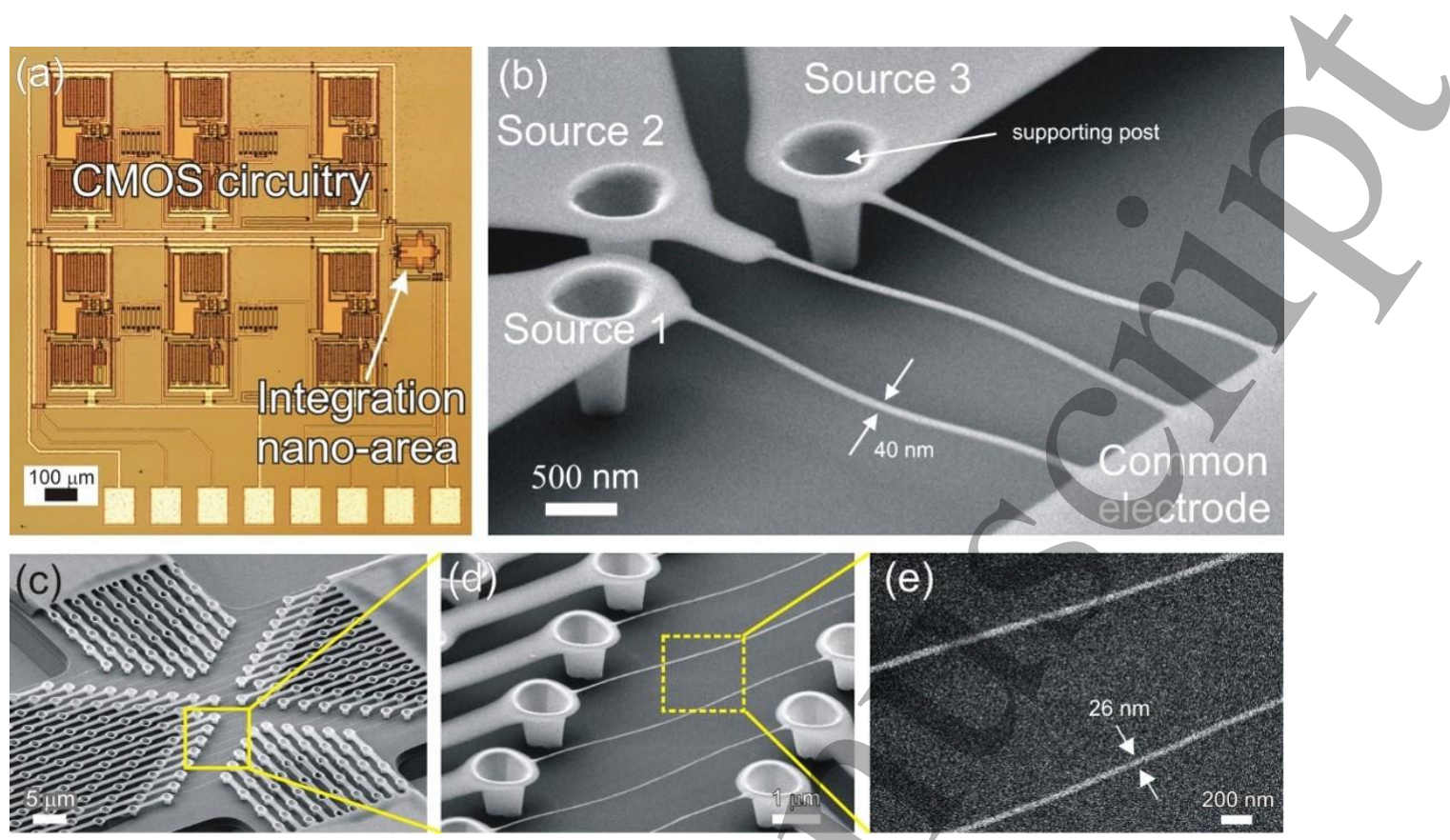

Figure 4. (a) Optical image of a complete CMOS circuit including the integration nano-area. (b) SEM image to show in high detail some suspended silicon beams and the microposts to sustain their connecting/contacting lines. (c-d) SEM images of another array of suspended silicon nanowires fabricated in an integration nano-area.

Figure 4 shows two different types of arrays fabricated in an integration nano-area. Figure 4 (a) is an optical image of the CMOS circuit attached to the integration area. Individually-addressable NWs (figure 4 (b)) and groups of NWs (figures 4 (c) to 4 (e)) have been fabricated. FIB irradiation is used to fabricate the whole structure, including the sub-micron lines connecting the circuit and the NWs (see figures 3 (c) and 4(c)). As the long connecting lines become also under-etched during the process to release the nanowires, they can collapse onto the substrate. To prevent the collapse of the fabricated structures, we have used a combination of FIB milling and FIB implantation to fabricate supporting micro-posts (see labelled figure 4 (b)). In terms of patterning resolution, suspended SiNWs having a diameter as small as $26 \mathrm{~nm}$ have been successfully integrated in the CMOS circuit (Figure 4 (e)).

The posts are defined at the same step (ion exposure) than the silicon nanowires. The area exposed to obtain a post corresponds to a circular pattern design. The post-shape fabrication is achieved by increasing the dosage applied in the center of the circular patterns, i.e. effective milling of silicon is produced in the center of the feature, while, at the same time, ion implantation occurs in the side-walls of the milled holes. The dose used to fabricate the pillars is $2.5 \times 10^{20}$ ions $/ \mathrm{cm}^{2}$. After wet etching, the side-walls of the posts remain because of their increase wet-etching resistance. As it can be observed in figures 4(b) and 4(d), the posts remain robustly attached to the silicon oxide layer that is located underneath the SOI layer. Additionally, the connecting lines are kept straight enough to correctly sustain the suspended nanowires.

Structurally, while the nanowires appear perfectly straight after the lithography process (figure 3 (b)), when they are released they tend to become curvy or bent. Distortion can be attributed to several causes, in particular, the presence of residual stress in the SOI layer in the starting wafer together with additional stress induced by the FIB implantation and annealing [46]. We have previously shown that the presence of this built-in asymmetries is necessary and actually favourable for obtaining an optimal transduction signal [2]. 
As for the effect of ion beam irradiation on CMOS electronics, we show that the fabrication process of the array will not induce damage such as to impede the proper circuit electronic operation needed to manage an array of integrated SiNWs. For the experimental assessment, we have used a specific test structure which is depicted in figure 5 (a). The structure consists of an integration nano-area located in close proximity to a NMOS transistor. Physical proximity and direct electrical connection implies that any potential effect of the fabrication process steps that may affect a CMOS circuit will also induce a change in the electrical characteristics of the NMOS transistor, which can be measured [27]. An electronic circuit operating as a multiplexing network consists of a set of transistors that are forced to commute adequately to provide a unique path to the selected nanowire. In consequence, it has to be assured that the whole commuting functionality of the transistors remains functional at the end of the fabrication process.
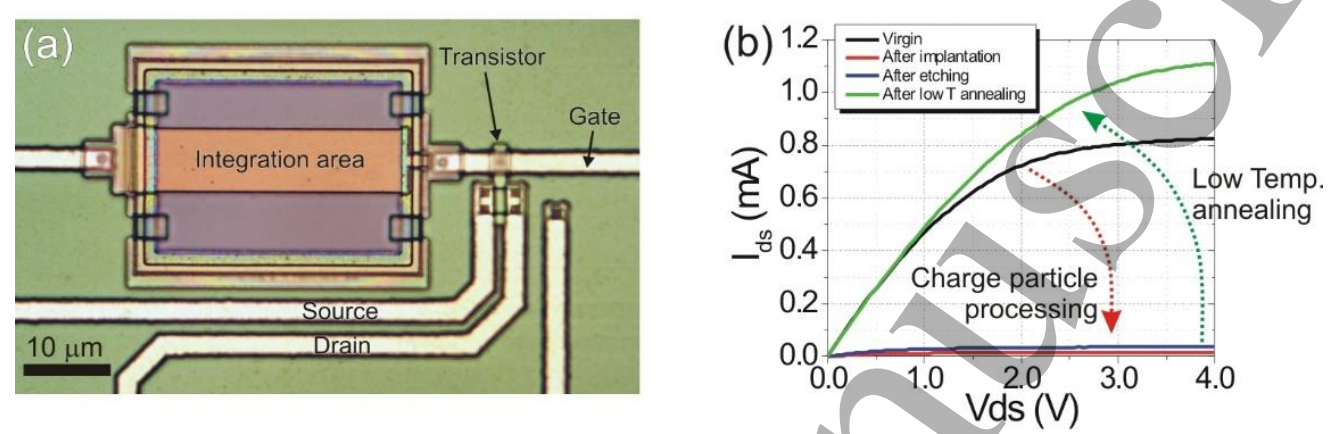

Figure 5. Optical image showing the test structure used to investigate the compatibility of the nanopatterning process: a NMOS transistor is located in close proximity to the integration area. S, D and G refers to the lines contacting the source, drain and gate of the MOS transistor, respectively (b) I/V curves of the NMOS transistors taken at different stages of the nanopatterning process.

Figure 5 (b) shows the electrical characteristics of the NMOS transistor after each one of the three steps used in the whole fabrication process: initial (after CMOS fabrication), after FIB irradiation (implantation), after etching and, finally, after a low temperature annealing $\left(300{ }^{\circ} \mathrm{C}\right)$. As it can be observed, the FIB lithography and etching steps prompts a drastic degradation of the electrical characteristics. The severe degradation is attributed to charge trapping that the generated secondary electrons cause in the gate of the MOS transistor by the incident ion beam [27] However, the electrical characteristics of the transistor can be recovered by applying a post-fabrication mild thermal treatment. After the annealing, charges are almost completely removed. Accordingly, we attribute the reversible degradation of the electric characteristics to a charge effect rather than to a physical damage of the transistor device layers. Yet, some effect does remain, as there are some differences with respect to the original I/V characteristics, which could be attributed to remaining charge trapping and incomplete recovery of Si material as a single crystal.

\section{Conclusions}

We have demonstrated the integration into CMOS circuits of arrays of suspended SiNWs being defined by FIB implantation, as a robust, simple and flexible nanopatterning method. We have shown that FIB implantation combined with $\mathrm{Si}$ wet etching provides advantages for the fabrication of mechanical resonators with coupled response, which is a result of an overhang that is formed during the same fabrication process. The electrical readout of the resulting nanowires system clearly shows the demonstration of coupled-modes operation. Importantly, coupled response can be precisely tuned by adjusting the separation between nanowires. 
Additional merit of the approach is represented by the fabrication of a large array of more than 3,000 silicon nanowires. It is implemented as a single exposure, and allows obtaining $100 \%$ fabrication yield. In terms of statistical patterning accuracy, the intrinsic size deviation of the process is small, for example, less than $10 \mathrm{~nm} \mathrm{SiNW}$ width dispersion is achieved.

Finally, we have proven that the process can be used to realize integrated micro/nanosystems based on the combination of CMOS circuits and arrays of suspended SiNWs. Their correct positioning in a dedicated area is successfully achieved. The array fabrication of the suspended nanowires relies on strategically incorporating micro-posts as a single FIB irradiation by adjusting exposure conditions. The electrical compatibility tests show that the ion-based (FIB) process does not produce any irreversible/ electrical/electronic damage to the CMOS circuit, demonstrating the processing feasibility for the integration of SiNWs mechanical resonators in CMOS circuits. 
[1] Voiculescu I and Zaghloul M 2015 Nanocantilever Beams: Modeling, Fabrication, and Applications. (CRC Press)

[2] Sansa M, Fernández-Regúlez M, Llobet J, San Paulo Á and Pérez-Murano F 2014 Highsensitivity linear piezoresistive transduction for nanomechanical beam resonators Nat. Commun. 54313

[3] Moser J, Eichler A, Güttinger J, Dykman M I and Bachtold A 2014 Nanotube mechanical resonators with quality factors of up to 5 million Nat. Nanotechnol. $91007-11$

[4] Craighead H G 2000 Nanoelectromechanical systems Science 290 1532-1535

[5] Ekinci K L and Roukes M L 2005 Nanoelectromechanical systems Rev. Sci. Instrum. 76 061101

[6] Gaspar J, Schmidt M E, Pedrini G, Osten W and Paul O 2010 Out-of-plane electrostatic microactuators with tunable stiffness Micro Electro Mech. Syst. MEMS IEEE 1131-4

[7] Verd J, Uranga A, Teva J, Lopez J L, Torres F, Esteve J, Abadal G, Perez-Murano F and Barniol N 2006 Integrated CMOS-MEMS with on-chip readout electronics for high-frequency applications IEEE Electron Device Lett. 27 495-7

[8] Loh O Y and Espinosa H D 2012 Nanoelectromechanical contact switches Nat. Nanotechnol. 7 283-95

[9] Husain A, Hone J, Postma H W C, Huang X M H, Drake T, Barbic M, Scherer A and Roukes M L 2003 Nanowire-based very-high-frequency electromechanical resonator Appl. Phys. Lett. 831240

[10] Sazonova V, Yaish Y, Üstünel H, Roundy D, Arias T A and McEuen P L 2004 A tunable carbon nanotube electromechanical oscillator Nature 431 284-287

[11] He R and Yang P 2006 Giant piezoresistance effect in silicon nanowires Nat. Nanotechnol. 1 $42-6$

[12] Lassagne B, Garcia-Sanchez D, Aguasca A and Bachtold A 2008 Ultrasensitive Mass Sensing with a Nanotube Electromechanical Resonator Nano Lett. 8 3735-8

[13] Chaste J, Eichler A, Moser J, Ceballos G, Rurali R and Bachtold A 2012 A nanomechanical mass sensor with yoctogram resolution Nat. Nanotechnol. 7 301-4

[14] Arcamone J, Rius G, Llobet J, Borrisé X and Pérez-Murano F 2008 Mass measurements based on nanomechanical devices: differential measurements J. Phys. Conf. Ser. 100052031 
[15] Verd J, Uranga A, Abadal G, Teva J, Torres F, Pérez-Murano F, Fraxedas J, Esteve J and Barniol N 2007 Monolithic mass sensor fabricated using a conventional technology with attogram resolution in air conditions Appl. Phys. Lett. 91013501

[16] Hanay M S, Kelber S, Naik A K, Chi D, Hentz S, Bullard E C, Colinet E, Duraffourg L and Roukes M L 2012 Single-protein nanomechanical mass spectrometry in real time Nat. Nanotechnol. 7 602-8

[17] Vidal-Álvarez G, Agustí J, Torres F, Abadal G, Barniol N, Llobet J, Sansa M, FernándezRegúlez M, Pérez-Murano F, San Paulo Á and Gottlieb O 2015 Top-down silicon microcantilever with coupled bottom-up silicon nanowire for enhanced mass resolution Nanotechnology 26145502

[18] Eom K, Park H S, Yoon D S and Kwon T 2011 Nanomechanical resonators and their applications in biological/chemical detection: Nanomechanics principles Phys. Rep. 503 115-63

[19] Cui Y, Wei Q, Park H and Lieber C M Nanowire Nanosensors for Highly Sensitive and Selective Detection of Biological and Chemical Species Science 293 1289-92

[20] Arlett J L, Maloney J R, Gudlewski B, Muluneh M and Roukes M L 2006 Self-Sensing Microand Nanocantilevers with Attonewton-Scale Force Resolution Nano Lett. 6 1000-6

[21] Dasgupta N P, Sun J, Liu C, Brittman S, Andrews S C, Lim J, Gaơ H, Yan R and Yang P 2014 25th Anniversary Article: Semiconductor Nanowires - Synthesis, Characterization, and Applications Adv. Mater. 26 2137-84

[22] Fernandez-Regulez M, Sansa M, Serra-Garcia M, Gil-Santos E, Tamayo J, Perez-Murano F and San Paulo A 2013 Horizontally patterned Si nanowire growth for nanomechanical devices Nanotechnology 24095303

[23] Kanda Y 1982 A graphical representation of the Piezoresistance Coefficients in Silicon IEEE Trans. Electron Devices 29 64-70

[24] Brugger J, Beljakovic G, Despont M, De Rooij N F and Vettiger P 1997 Silicon micro/nanomechanical device fabrication based on focused ion beam surface modification and KOH etching Microelectron. Eng. 35 401-404

[25] Llobet J, Sansa M, Gerbolés M, Mestres N, Arbiol J, Borrisé X and Pérez-Murano F 2014 Enabling electromechanical transduction in silicon nanowire mechanical resonators fabricated by focused ion beam implantation Nanotechnology 25135302

[26] Llobet J, Sansa M, Lorenzoni M, Borrisé X, San Paulo A and Pérez-Murano F 2015 Tuning piezoresistive transduction in nanomechanical resonators by geometrical asymmetries Appl. Phys. Lett. 107073104

[27] Rius G, Llobet J, Arcamone J, Borrisé X and Pérez-Murano F 2009 Electron- and ion-beam lithography for the fabrication of nanomechanical devices integrated on CMOS circuits Microelectron. Eng. 86 1046-9

[28] Chekurov N, Grigoras K, Sainiemi L, Peltonen A, Tittonen I and Franssila S 2010 Dry fabrication of microdevices by the combination of focused ion beam and cryogenic deep reactive ion etching J. Micromechanics Microengineering 20085009

[29] Sharma A, Suma B N, Bhat K N and Naik A K 2017 Gallium-Doped Piezoresistive Sensor With Optimized Focused Ion Beam Implantation J. Microelectromechanical Syst. 26 127-34 
[30] La Marche P H 1983 Focused ion beam microlithography using an etch-stop process in gallium-doped silicon J. Vac. Sci. Technol. B Microelectron. Nanometer Struct. 11056

[31] Choquette K D and Harriott L R 1993 Dry lithography using focused ion beam implantation and reactive ion etching of $\mathrm{SiO} 2$ Appl. Phys. Lett. 623294

[32] Zachariasse J M and Walker J F 1997 Direct write patterning of titanium films using focused ion beam implantation and plasma etching Microelectron. Eng. 35 63-66

[33] McKenzie W, Pethica J and Cross G 2011 A direct-write, resistless hard mask for rapid nanoscale patterning of diamond Diam. Relat. Mater. 20 707-10

[34] Liu Z, Iltanen K, Chekurov N, Grigoras K and Tittonen I 2013 Aluminum oxide mask fabrication by focused ion beam implantation combined with wet etching Nanotechnology 24 175304

[35] Giannuzzi, L A and Stevie F A 2004 Introduction to Focused Ion Beams (Springer)

[36] Rius G, Llobet J, Borrisé X, Mestres N, Retolaza A, Merino S and Perez-Murano F 2009 Fabrication of complementary metal-oxide-semiconductor integrated nanomechanical devices by ion beam patterning J. Vac. Sci. Technol. B Microelectron. Nanometer Struct. 272691

[37] Ziegler J F, Ziegler M D and Biersack J P 2010 SRIM - The stopping and range of ions in matter (2010) Nucl. Instrum. Methods Phys. Res. Sect. B Beam Interact. Mater. At. 268 1818-23

[38] Jordi Llobet 2016 Focused ion beam implantation as a tool for the fabrication of nano electromechanical devices (Universitat Autònoma de Barcelona)

[39] Tokoro K, Uchikawa D, Shikida M and Sato K 1998 Anisotropic etching properties of silicon in KOH and TMAH solutions Micromechatronics and Human Science, 1998. MHS'98. Proceedings of the 1998 International Symposium on (IEEE) pp 65-70

[40] Llobet J, Gerbolés M, Sansa M, Bausells J, Borrisé X and Pérez-Murano F 2015 Fabrication of functional electromechanical nanowire resonators by focused ion beam implantation $J$ MicroNanolith MEMS MOEMS 14031207

[41] Llobet J, Krali E, Wang C, Arbiol J, Jones M E, Pérez-Murano F and Durrani Z A K 2015 Resonant tunnelling features in a suspended silicon nanowire single-hole transistor Appl. Phys. Lett. 107223501

[42] Gil-Santos E, Ramos D, Pini V, Calleja M and Tamayo J 2011 Exponential tuning of the coupling constant of coupled microcantilevers by modifying their separation Appl. Phys. Lett. 98123108

[43] Giner J, Uranga A, Muñóz-Gamarra J L, Marigó E and Barniol N 2012 A fully integrated programmable dual-band RF filter based on electrically and mechanically coupled CMOSMEMS resonators J. Micromechanics Microengineering 22055020

[44] Gil-Santos E, Ramos D, Jana A, Calleja M, Raman A and Tamayo J 2009 Mass Sensing Based on Deterministic and Stochastic Responses of Elastically Coupled Nanocantilevers Nano Lett. 9 4122-7

[45] Villarroya M, Figueras E, Montserrat J, Verd J, Teva J, Abadal G, Murano F P, Esteve J and Barniol N 2006 A platform for monolithic CMOS-MEMS integration on SOI wafers $J$. Micromechanics Microengineering 16 2203-10 
[46] Lorenzoni M, Llobet J, Gramazio F, Sansa M, Fraxedas J and Perez-Murano F 2016 Evaluating the compressive stress generated during fabrication of Si doubly clamped nanobeams with AFM J. Vac. Sci. Technol. B Nanotechnol. Microelectron. Mater. Process. Meas. Phenom. 34 06KK02 